\title{
Factors Associated with Antigen HBs Seroconversion among Blood Donors in Ouagadougou from 2008 to 2017
}

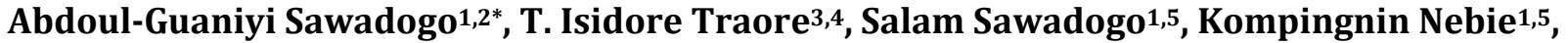 \\ Lucien Désiré Dahourou ${ }^{6}$, Juste Some ${ }^{7}$, Hervé Kpoda ${ }^{3}$, Ahmed Kabore ${ }^{5}$, Dahourou Honorine ${ }^{1}$, \\ Arzouma Paul Yooda1, Bia Emile Drabo', Jean-Etienne Koanda', Alain Konseybo', \\ Eléonore Kafando5, Nicolas Meda ${ }^{3,5}$, Léon Blaise Savadogo ${ }^{3,4}$
}

${ }^{1}$ Centre National de Transfusion Sanguine (CNTS), Ouagadougou, Burkina Faso

${ }^{2} \mathrm{ONG}$ Terre des hommes Lausanne, Ouagadougou, Burkina Faso

${ }^{3}$ Centre MURAZ, Bobo-Dioulasso, Burkina Faso

${ }^{4}$ Université Nazi BONI, Bobo-Dioulasso, Burkina Faso

${ }^{5}$ Université Joseph KI-ZERBO, Ouagadougou, Burkina Faso

${ }^{6}$ Institut de Recherche en Sciences de la Santé (IRSS), Ouagadougou, Burkina Faso

${ }^{7}$ Université Norbert ZONGO, Koudougou, Burkina Faso

Email: *lesage2004bk@yahoo.fr

How to cite this paper: Sawadogo, A.-G. Traore, T.I., Sawadogo, S., Nebie, K., Dahourou, L.D., Some, J., Kpoda, H., Kabore, A., Honorine, D., Yooda, A.P., Drabo, B.E., Koanda, J.-E., Konseybo, A., Kafando, E., Meda, N. and Savadogo, L.B. (2021) Factors Associated with Antigen HBs Seroconversion among Blood Donors in Ouagadougou from 2008 to 2017. Advances in Infectious Diseases, 11, 278-289.

https://doi.org/10.4236/aid.2021.113025

Received: June 4, 2021

Accepted: September 3, 2021

Published: September 6, 2021

Copyright $\odot 2021$ by author(s) and Scientific Research Publishing Inc. This work is licensed under the Creative Commons Attribution International License (CC BY 4.0).

http://creativecommons.org/licenses/by/4.0/

(c) (i) Open Access

\begin{abstract}
Introduction: The risk of transmission of pathogens such as hepatitis B virus threatens the safety of transfused patients especially in high endemic areas. The aim of this study was to determine the incidence and factors associated with hepatitis B virus surface antigen (HBsAg) seroconversion in blood donors at the Regional Blood Transfusion Centre of Ouagadougou. Methods: A retrospective cohort study of voluntary non-remunerated blood donors (VNRBD), was conducted from 2008 to 2017. Data on HBsAg seroconversion were collected. The Kaplan-Meier method and the Log-Rank test were used to estimate the survival curves. Cox's regression identified the factors associated with this seroconversion. Results: Of 23,494 donors, 559 had HBsAg seroconversion. The number of donor years was 58,637.50 and the HBV incidence rate was 9.53 per 1000 donor years. The median seroconversion time was 75.73 months with extremes of 2.7 months and 107.12 months. The risk of seroconversion was 1.30 times higher among donors aged 21 to 24 years old $(\mathrm{p}=0.007)$ and 2.49 times higher among those over 24 years old $(\mathrm{p}<$ $0.0001)$ than among donors under 21 years old. Female donors were 1.11 times more likely to seroconvert than male donors $(p=0.33)$. Donor residence was not significantly associated with HBsAg seroconversion (Hazard
\end{abstract}


ratio $=1.12 ; \mathrm{p}=0.36)$. The risk of seroconversion decreased significantly with the number of blood donations (Hazard ratio $=0.58 ; \mathrm{p}=0.006$ ). Conclusion: The incidence of HBsAg remains high among blood donors, which could have a negative impact on transfusion safety. The age of the blood donor was significantly associated to AgHBs seroconversion.

\section{Keywords}

Time to Onset, Viral Hepatitis B, Blood Donors, Seroconversion

\section{Introduction}

Blood transfusion safety, despite the progress made particularly in the prevention of infectious and immunological risk, remains a concern especially in developing countries. Although screening of all blood donations for certain transfusion-transmissible infections including hepatitis B virus (HBV) has become routine in almost all countries, the risk of transmitting infectious agents through blood transfusion is not yet fully mastered [1] [2]. This residual risk of transmission of infectious agents by blood transfusion is mainly related to the serological window period, a period between onset of infection and the appearance of markers detectable with tests. HBV is one of the infectious agents whose transmission by transfusion is feared and more likely due to a relatively wide silent period (56 days on average) [3].

Burkina Faso is located in an area of high endemicity of HBV infection with a prevalence of $9.1 \%$ in the general population [4]. Data from the National Blood Transfusion Center (NBTC) shows that the positivity rate of HBs surface antigen (HBsAg) in donated blood remains high over the past three years: $8.55 \%$ in 2015, then $8.66 \%$ in 2016 and $8.95 \%$ in 2017 . This rate was $0.93 \%$ in 2017 among repeated blood donors [5] [6], meaning that about one in one hundred donors go from $\mathrm{HBsAg}$ negative to $\mathrm{HBsAg}$ positive, thus impacting transfusion safety.

The occurrence of HBsAg seroconversion indicates exposure to the risk of HBV infection. Several factors may be associated with this exposure, and therefore, with HBsAg seroconversion. The aim of this study was to determine the socio-demographic factors associated with the seroconversion of $\mathrm{HBsAg}$ in blood donors aged 18 to 27 at the Regional Blood Transfusion Center of Ouagadougou (RBTC/O).

\section{Methods}

\subsection{Site and Period of Study}

The study was carried out at the RBTC/O which includes two fixed blood donation sites, the Paspanga site at Ouagadougou and the Tengandgo site in the rural commune of Komsilga.

The study covered the period from January 1, 2008 to December 31, 2017. 
Data was collected in March 2019.

\subsection{Study Design}

The selection of participants was based on an exhaustive sampling of all blood donors aged 18 to 27 years registered in the Medicotechnical Software MTS INLOG $^{\odot}$ database of the RBTC/O who had made at least two blood donations during the study period and in whom the first blood donation was tested negative for the four markers (HIV, HBV, HCV and Syphilis).

This choice takes into account the blood donors loyalty policy in force at the National Blood transfusion center (NBTC) through the promotion of "Clubs 25 ". This is a group of blood donors aged between 18 and 27 who undertake to give blood regularly, at least 25 times before their 27th birthday, to adopt behaviors that reduce the risk of contamination by infections transmissible by blood transfusion and to promote blood donation around them.

Since November 2016, the consent of blood donors is systematically obtained before any blood donation by signing the pre-donation medical interview questionary form.

The variables studied were the serological result of HBsAg (positive or negative) for the dependent variable and age, sex, place of residence (urban or rural) and the number of blood donations for the independent variables.

\subsection{Data Extraction and Processing}

The data was extracted from the MTS INLOG ${ }^{\circ}$ blood donors' management database. A SQL (Structure Querry Language) computer query, including the variables of interest as well as the "Donor" identifier and blood donation numbers, enabled the data to be extracted and exported to an Excel file version 2013 for processing and clearance. Aberrant or missing data and donors not meeting the inclusion criteria have been removed (Figure 1).

\subsection{Operational Definitions and Calculation Formulas}

Survival function: it's the probability for each blood donor to remain seronegative each time an HBsAg seroconversion occurs and this until the date of the latest news or until December 31, 2017, the date of end of our study. Otherwise, it is the proportion of blood donors who did not seroconvert during the follow-up period.

Incidence rate (IR) of HBsAg seroconversion is the "production rate" of new cases, i.e. of blood donors who were tested positive for HBsAg after negative tests in the past time. It is therefore equal to the number of new cases per unit of time divided by the size of the population. The population size is calculated in donor years. The incidence rate is therefore calculated by dividing the number of new HBsAg positive cases by the number of donor years.

Donor-years (D-Y): it's the sum of the cumulative durations in years over the entire study population, during which donors are likely to be registered as new positive HBsAg cases. 


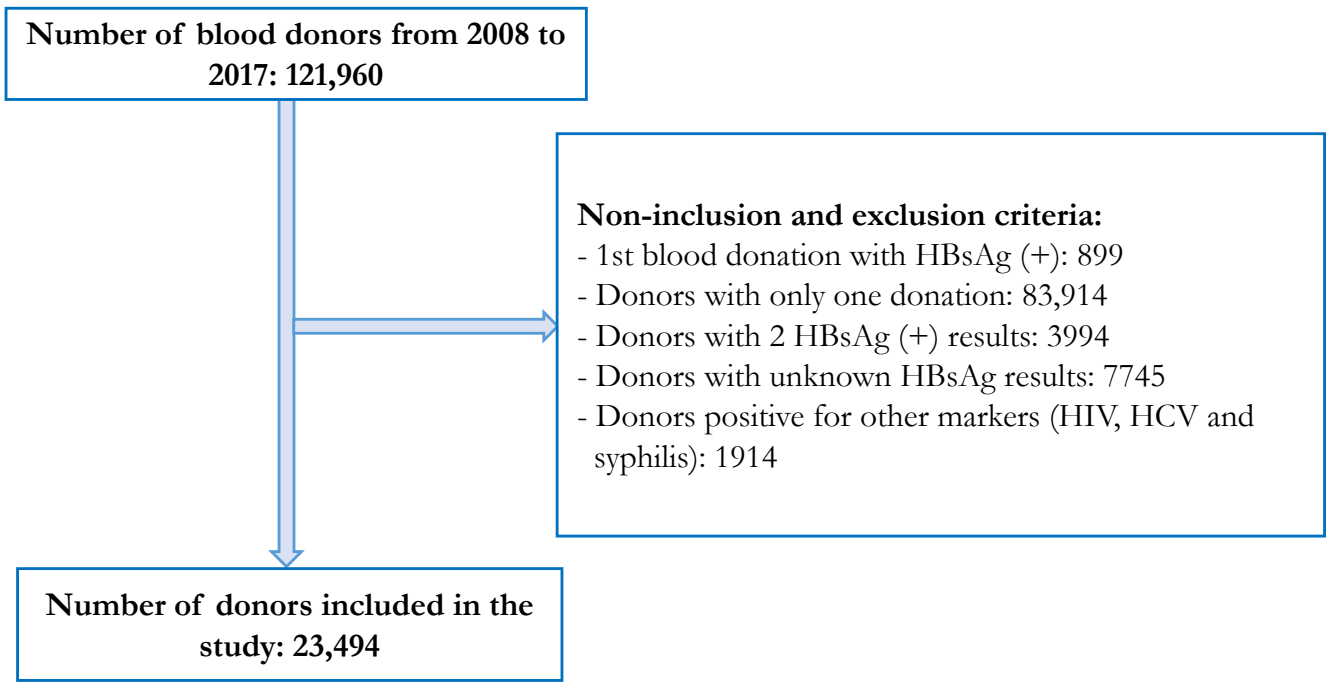

Figure 1. Flow chart of blood donors selection for the study at regional blood transfusion center of Ouagadougou, from 2008 to 2017.

Cumulative Incidence (CI): is the ratio of the number of new HBsAg-positive cases (in the general population or by specific group) during the follow-up period of blood donors divided by the population of blood donors at risk of becoming HBsAg-positive.

Median survival time: it's the time within which half of the blood donors in our sample become HBsAg positive, the other half still being seronegative to HBsAg. The chance of a randomly selected individual becoming HBsAg negative before this time frame is reached is $50 \%$.

\subsection{Data Analysis}

Data analysis had been performed using STATA SE 15 software. To account for missing data, lost (premature renouncement of blood donation), an intention-to-treat analysis was applied.

For each variable, descriptive statistics were produced. The Pearson Chi-2 test was used to compare proportions.

The Kaplan-Meier estimator was used to determine the median time of occurrence of HBsAg seroconversions, to estimate survival curves and to define the probabilities of HBsAg seroconversion after the first blood donation. Verification of the equality of median times between the different groups of blood donors was done using the Log-Rank Test.

In multivariate analysis, the top-down "step-by-step" selection method was used to reduce the model. The overall adequacy of the multivariate model was checked by calculating Cox-Snell residuals through a graphical representation of the Nelson-Aalen cumulative risk function and the Cox-Snell residuals.

The Cox model was used to determine the relationships between the risk function associated with the occurrence of HBsAg seroconversion and the explanatory variables. The significance level was set at $\mathrm{p}<0.05$ for all analyses. 


\section{Results}

\subsection{Sociodemographic Characteristics of Blood Donors}

The study concerned 23,494 blood donors of whom $71.23 \%$ were male and $85.75 \%$ lived in urban areas. The average age of blood donors at their first donation was 21.06 years $[18 ; 26.83$ years]. The highest number of blood donations made by a donor was 33 donations and $7.75 \%$ of donors had made at least 10 blood donations (Table 1).

A total of 559 (2.38\%) of the 23,494 blood donors seroconverted HBsAg. The mean age at the time of seroconversion was 23.10 years [18.42; 27] and the majority (475 donors), lived in urban areas.

\subsection{Incidence of HBsAg Seroconversion}

The number of donor years was 58,637.49 and the incidence rate of HBsAg was 9.53 cases per $1000 \mathrm{D}$-Y. Table 2 shows the incidence rates of HBsAg by age, gender and place of residence.

Table 1. Socio-demographic characteristics of the RBTC/O blood donors who seroconverted to HBsAg $(\mathrm{n}=559)$ between 2008 and 2017.

\begin{tabular}{ccc}
\hline Socio-demographic characteristics & Effective & Pourcentage (\%) \\
\hline Ages groups (years) & & \\
\hline$[18-21[$ & 143 & 25.58 \\
{$[21-24[$} & 217 & 38.82 \\
{$[24-27]$} & 199 & 35.60 \\
\hline Gender & & \\
\hline Male & 435 & 77.82 \\
Female & 124 & 22.18 \\
\hline Place of residence & & \\
\hline Urban & 84 & 15.03 \\
Rural & 475 & $\mathbf{8 4 . 9 7}$ \\
\hline
\end{tabular}

Table 2. Distribution of incidence rates and median survival times by blood donors $(n=559)$ characteristics at RBTC/O from 2008 to 2017.

\begin{tabular}{cccc}
\hline Characteristics & Donors-years (D-Y) & Incidence rate (per 1000 D-Y) & Median survival time (months) \\
\hline Gender & & & \\
\hline Male & $47,747.79$ & 9.11 & 76.9 \\
Female & $10,889.69$ & 11.39 & 73.63 \\
\hline Ages groups & & & \\
\hline$[18-21[$ & $37,962.89$ & 8.19 & 80.30 \\
{$[21-24[$} & $17,609.89$ & 10.11 & 55.60 \\
{$[24-27]$} & 3064.69 & 22.84 & 27.63 \\
\hline Place of residence & & & 76.53 \\
\hline Urban & $52,104.29$ & 9.12 & 61.76 \\
Rural & 6533.19 & 12.86 & 75.73 \\
Total & $58,637.499$ & 9.53 & \\
\hline
\end{tabular}


The incidence of HBV was 2.58\% (311/12,046), 2.13\% (178/8345) and 2.25\% (70/3103) respectively in donors under 21 years old, 21 to under 24 and 24 years old and over $(\mathrm{p}<0.001$; Chi $2=70.05)$. Among male donors, 435 out of 16,735 (2.60\%) incident cases of HBV were recorded compared to 124 out of 6759 among female donors ( $\mathrm{p}=0.024$; Chi2 $=5.08$ ).

According to the place of residence, the incidence of $\mathrm{HBV}$ was $2.36 \%$ $(475 / 20,147)$ among blood donors residing in urban areas compared to $2.51 \%$ $(34 / 3347)$ among those residing in rural areas $(\mathrm{p}=0.0068$; Chi2 $=7.33)$.

\subsection{Estimation of the Survival Function of AgHBs Seroconversion}

The overall survival function was $90.13 \%$ at one year and dropped to $57.96 \%$ at 5 years. The shortest HBsAg seroconversion time was 2.7 months and the longest was 107.12 months. The median survival time (the time after which $50 \%$ of HBsAg seroconversions have already occurred during the follow-up period) was 75.73 months (Figure 2).

The survival function was $90.98 \%$ at one year and dropped to $63.83 \%$ at 5 years in donors aged 18 to less than 21 years, $92.38 \%$ at one year, $41.37 \%$ at 5 years in those aged 21 to less than 24 years and $78.54 \%$ at one year, dropping to $17.05 \%$ at 5 years in blood donors who were at least 24 years old. The log-rank test was 70.04 and p-value $<0.0001$ (Figure 3).

\subsection{Factors Associated with HBsAg Seroconversion}

The risk of seroconversion was statistically related to the donor's age with a 1.30 and 2.49 times higher risk respectively for blood donors between 21 and under 24 years old $(\mathrm{p}=0.007)$ and over 24 years old $(\mathrm{p}<0.001)$ compared to donors under 21 years old. The risk of seroconversion decreased among blood donors from the 10th blood donation onwards (Table 3).

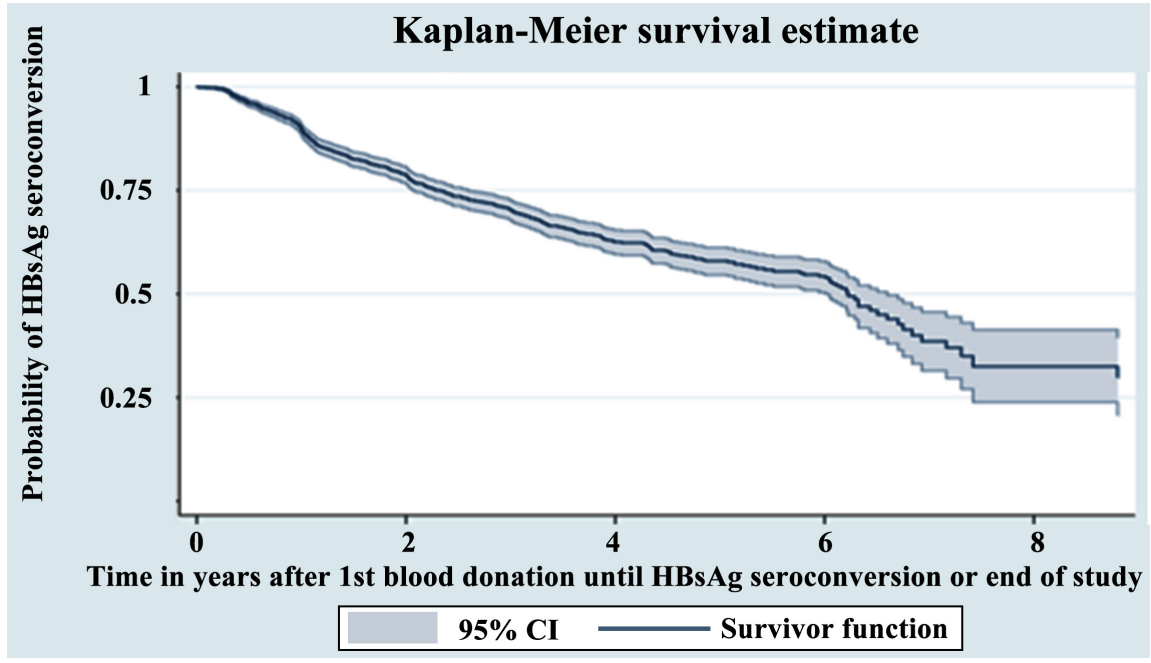

Figure 2. Kaplan-Meier survival function plot of HBsAg-related seroconversion in blood donors from 2008 to 2017 at regional blood transfusion center of Ouagadougou, from 2008 to 2017. 


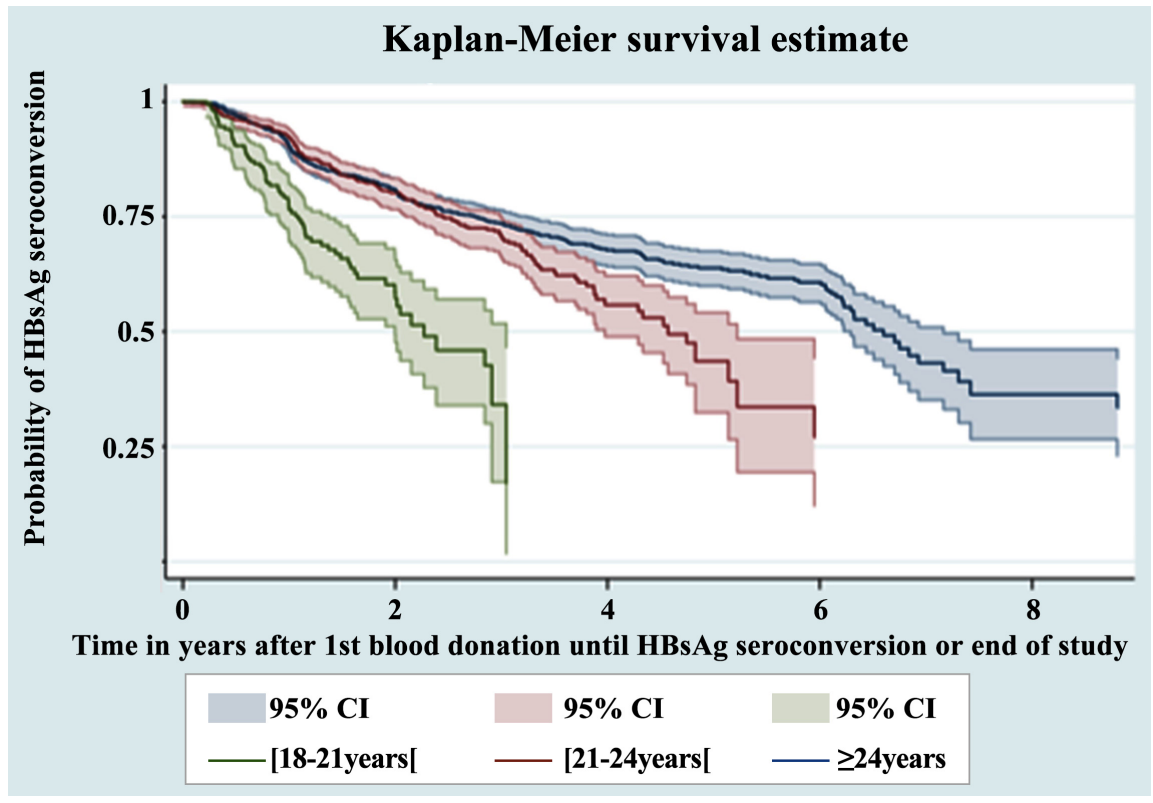

Figure 3. Kaplan-Meier survival function plot of HBV-related seroconversion by donor age at first donation from 2008 to 2017 at regional blood transfusion center of Ouagadougou.

Table 3. Cox multivariate regression model applied to the effect of blood donors $(\mathrm{n}=$ 559) gender, age, residence, and number of donations on HBsAg seroconversion among blood donors at RBTC/O from 2008 to 2017.

\begin{tabular}{|c|c|c|c|}
\hline $\begin{array}{l}\text { Socio-demographics } \\
\text { characteristics }\end{array}$ & Hazard ratio (HR) & p-value & $\begin{array}{c}\text { Confidence } \\
\text { interval (CI) } 95 \%\end{array}$ \\
\hline \multicolumn{4}{|l|}{ Gender of blood donors } \\
\hline Male & 1 & - & - \\
\hline Female & 1.108 & 0.325 & [0.903 - 1.359] \\
\hline \multicolumn{4}{|l|}{ Ages groups (years) } \\
\hline$[18-21[$ & 1 & - & - \\
\hline$[21-24[$ & 1.302 & 0.007 & {$[1.074-1.579]$} \\
\hline 24 et plus & 2.485 & $<0.001$ & {$[1.893-3.262]$} \\
\hline \multicolumn{4}{|l|}{ Place of residence } \\
\hline Urban & 1 & - & - \\
\hline Rural & 1.118 & 0.362 & {$[0.879-1.421]$} \\
\hline \multicolumn{4}{|c|}{ Number of blood donations } \\
\hline$[2-9]$ & 1 & - & - \\
\hline [10 - 19] & 0.583 & 0.006 & {$[0.397-0.858]$} \\
\hline$[20-25]$ & - & - & - \\
\hline$>25$ & 0.387 & 0.344 & {$[0.054-2.765]$} \\
\hline
\end{tabular}

Log Rank Test $=1774.48 ;$ p-value $<0.0001$. 


\section{Discussion}

Our study determined that the incidence rate of HBV infection in blood donors was 9.53 cases per $1000 \mathrm{D}$-Y. The median seroconversion time was 75.73 months with a 2.5 times higher risk of seroconversion in donors over 24 years old compared to those under 21 years old $(\mathrm{p}<0.0001)$. This risk decreased significantly with the number of blood donations made.

This study is the first of its kind among blood donors in Burkina Faso. It provides information on the risk of HBsAg seroconversion and associated socio-demographic factors. This can guide blood donors selection policy at the NBTC. However, our study has some limits. First, the failure to take into account certain potentially explanatory variables such as the site of blood donation, level of education, socioeconomic level, occupation, medical background, etc. This can limit the exact knowledge of the range of risk factors that could be associated with HBsAg seroconversion. In addition, the high number of people lost to follow-up (early renouncement of blood donation), the lack of confirmatory testing of HBsAg serological results, and the transient nature of HBsAg in blood (seroconversion to HBsAg and subsequent recovery prior to blood donation) may have limited the extent of testing.

\subsection{Incidence of HBsAg Seroconversion}

Among the 23,494 blood donors, 559 seroconversions to the HBsAg were observed, for a cumulative incidence of $2.38 \%$. The incidence rate was 9.53 per $1000 \mathrm{D}-\mathrm{Y}$ and increased with the age of the blood donors. These results are higher than those of YOODA et al. [3] who reported 316 cases (1.99\%) of seroconversions to HBsAg and NAGALO et al. [7] who reported $1.66 \%$ in their studies covering the periods 2015-2017 (i.e. 3 years) and 2009 respectively. In the Democratic Republic of Congo (DRC), KABINDA et al. [8] found an incidence of 2.05\% (37/1804) over the period 2010-2012, results close to ours. Both the YOODA et al. and NAGALO et al. studies were conducted in the same blood transfusion centre as ours, but included a wider age range of donors (17 - 65 years) than in our study (18 - 27 years), which partly explains the disparities found.

Indeed, the blood donors included in our study were all closer in age to adolescents' first sexual experiences, which occur in a context of low levels of knowledge about sexuality and sexual and reproductive health, leading to risky behaviors and practices [9].

In addition, the broader time span of our study (10 years compared to three and one year in the other two studies) could be another explanation, in that the longer the follow-up time, the higher the risk of seroconversion. Furthermore, our study included a larger study population with 1.5 times and 5 times that of YOODA et al. and NAGALO et al. respectively.

Even, the low incidences observed in the other two studies would be due to constant efforts to raise awareness and popularise vaccination against the hepati- 
tis $B$ virus during the last decade.

The incidence rate of HBV infection among blood donors in our study was 9.53 HBsAg seroconversions per $1000 \mathrm{D}-\mathrm{Y}$. This rate was lower than that found by NAGALO et al. [10] which was 50.39 per $1000 \mathrm{D}-\mathrm{Y}$ in 2009 in a study conducted in three regional blood transfusion centers in Burkina Faso and that found by TOURE-FALL et al. [11] with 66.78 per 1000 D-Y between 2003 and 2005 in Dakar, Senegal. These results could be attributed to the difference in seroprevalence of viral hepatitis B both between and within countries but also between age groups of blood donors and to screening techniques for HBsAg used on blood donations.

However, this incidence is much higher than that observed in France by PILLONEL et al. [12] who reported an incidence rate of 0.72 per 100,000 D-Y for HBV between 2008 and 2010 among blood donors. These data reflect the strong differences in hepatitis B seroprevalence between France (low HBV endemicity area) and Burkina Faso (high HBV endemicity area), the effectiveness of medical screening of blood donors (performed by medical personnel in France) and differences in HBsAg screening techniques.

Improving blood transfusion safety in a context of high endemicity of HBV infection requires the implementation of new blood donors recruitment strategies to recruit and retain lower-risk donors, improve the pre-donation medical selection of blood donors, and vaccinate the entire population, particularly those of age to give blood, in order to maintain a sufficient pool of "safe" blood donors.

\subsection{Factors Associated with HBsAg Seroconversion in Blood Donors}

The instantaneous risk or Hazard ratio (HR) of becoming HBsAg-positive was 1.302 times higher among blood donors aged 21 to less than 24 years old $(\mathrm{p}=$ 0.007; CI [21; 24]: [1.074; 1.579]) and 2.485 times higher among those aged 24 years old and older ( $\mathrm{p}<0.001$; IC $\geq 24$ ans: [1.893; 3.262]).

These results are similar to those of NAGALO et al. [13] who found a risk of 1.48 times higher $(\mathrm{p}=0.015)$ increased risk $(\mathrm{p}=0.015)$ among donors aged 20 29 years and 1.30 -fold $(\mathrm{p}=0.127)$ among blood donors under 20 years of age.

Our results are similar to those of NAGALO et al. [13] who found a risk of 1.48 times higher $(\mathrm{p}=0.015)$ in donors aged 20 to 29 and 1.30 times $(\mathrm{p}=0.127)$ in blood donors under 20 years.

The instantaneous risk or Hazard ratio (HR) of becoming HBsAg-positive was 1.302 times higher among blood donors aged 21 to less than 24 years old ( $\mathrm{p}=$ 0.007 ; CI $[21 ; 24]:[1.074 ; 1.579])$ and 2.485 times higher among those aged 24 years old and older ( $<<0.001$; IC $\geq 24$ ans: [1.893; 3.262]). These results are similar to those of NAGALO et al. [13] who found a risk of 1.48 times higher ( $p$ $=0.015)$ among donors aged 20 to 29 years old and 1.30 times $(\mathrm{p}=0.127)$ among blood donors under 20 years old. 
This could be explained by the persistence or even intensification of the adoption of risky behaviors over time. These results would also suggest that the adoption of sexual behaviors at risk is more frequent with age because of financial independence or autonomy with the acquisition of stable employment and more consistent income.

Awareness-raising must be continuous on actions to prevent HBV infections such as strict compliance with universal hygiene measures, disinfection and sterilization measures for blunt, sharp and cutting materials, the use of single-use materials, sexual education and especially vaccination against $\mathrm{HBV}$.

The risk of HBsAg seroconversion was 1.108 times higher for female donors compared to male donors, but this difference was not statistically significant $(\mathrm{p}=$ 0.325). Our results are confirmed by those of PILLONEL et al. [12] in France and MWENEBITU et al. in the DRC [14] who found no gender difference in HBV infection. These results would suggest that male blood donors are as exposed to HBV infection as female blood donors.

In contrast, the results of the NAGALO et al. study [13] in Burkina Faso in 2009 reported a statistically significant difference (Odds ratio $=1.47 ; \mathrm{p}<0.001$ ) in the occurrence of HBV infection in male versus female blood donors. Actions for the prevention and management of blood transfusion-transmitted infections should therefore concern both men and women.

Regarding the place of residence of blood donors, no significant association was found between urban and rural blood donors in the occurrence of HBsAg seroconversion $\left(\mathrm{HR}_{\text {rural }}=1.118 ; \mathrm{p}=0.362\right)$. Unlike our study, NAGALO et al. [13] found a higher risk in rural areas (Odds ratio $=1.54 ; \mathrm{p}<0.001$ ).

These results prove that hepatitis $B$ affects both urban and rural areas. The involvement of administrative authorities, opinion leaders, religious and customary leaders, youth and women's associations in the promotion of blood donation and the adoption of lower-risk behaviors could strengthen the adequate and regular supply of safe blood products.

Finally, the risk of becoming HBsAg-positive among blood donors decreased with the number of blood donations (Hazard ratio $=0.583 ; \mathrm{p}=0.006$ ). This finding has been made in the literature, supporting the idea that loyal and regular blood donors are less at risk of transfusion-transmissible infections [14]. The emphasis should then be on recruiting and especially retaining blood donors in order to meet the needs for quality blood.

This study showed the increase in the age of blood donors was significantly associated with the incidence of viral hepatitis B among blood donors, while increasing the number of blood donations among blood donors decreased the risk of becoming HBsAg positive.

\section{Conclusions}

The incidence rate of HBV infection remains high among blood donors with 9.53 seroconversions per $1000 \mathrm{D}-\mathrm{Y}$. The $\mathrm{HBsAg}$ seroconversion is statistically 
associated with the increase in age of blood donors, but decreased with the number of blood donations.

Continuous improvement in blood transfusion safety is a concern for health authorities and blood transfusion staff. It requires the recruitment and retention of "safe" blood donors, their accountability and education.

The implementation of blood donors' vaccination program would reduce the incidence of HBV infection among blood donors and increase the availability of blood products. Further studies on other risk factors, factors associated with HBV infection, and behavioral studies of blood donors could allow a better description of viral hepatitis B among blood donors in Burkina Faso.

\section{Acknowledgements}

Special thanks to Mr. LINGANI Cheick Ali for his contribution in data extraction.

\section{Conflicts of Interest}

The authors declare no conflicts of interest regarding the publication of this paper.

\section{References}

[1] Laperche, S. and Lefrère, J.-J. (2011) Blood-Borne Agents and Transfusion of Blood Products. Hématologie, 17, 225-236. https://doi.org/10.1684/hma.2011.0595

[2] Uwingabiye, J., Zahid, H., Unyendje, L., et al. (2016) Séroprévalence des marqueurs viraux sur les dons du sang au Centre de Transfusion Sanguine, Hôpital Militaire d'Instruction Mohammed V de Rabat. Pan African Medical Journal, 25, 5. https://doi.org/10.11604/pamj.2016.25.185.6266

[3] Yooda, A.P., Sawadogo, S., Soubeiga, S.T., et al. (2019) Residual Risk of HIV, HCV, and HBV Transmission by Blood Transfusion between 2015 and 2017 at the Regional Blood Transfusion Center of Ouagadougou, Burkina Faso. Journal of Blood Medicine, 10, 1-6. https://doi.org/10.2147/JBM.S189079

[4] Meda, N., Tuaillon, E., Kania, D., et al. (2018) Hepatitis B and C Virus Seroprevalence, Burkina Faso: A Cross-Sectional Study. Bulletin of the World Health Organization, 96, 750-759. https://doi.org/10.2471/BLT.18.208603

[5] Ministère de la santé. Centre national de transfusion sanguine, Burkina Faso (2018) Annuaire statistique de la transfusion sanguine 2017. CNTS, Ouagadougou.

[6] Ministère de la santé-Centre national de transfusion sanguine, Burkina Faso (2019) Annuaire statistique de la transfusion sanguine 2018. CNTS, Ouagadougou.

[7] Nagalo, B.M., Bisseye, C., Sanou, M., et al. (2012) Seroprevalence and Incidence of Transfusion-Transmitted Infectious Diseases among Blood Donors from Regional Blood Transfusion Centres in Burkina Faso, West Africa: Transfusion-Transmitted Infections in Burkina Faso. Tropical Medicine \& International Health, 17, 247-253. https://doi.org/10.1111/j.1365-3156.2011.02902.x

[8] Kabinda, J.M., Bulabula, A.N., Donnen, P., et al. (2014) Residual Risk of Transmission of HIV and Hepatitis B and C by Blood Transfusion in Bukavu in the Democratic Republic of Congo. Open Journal of Epidemiology, 4, 157-163. https://doi.org/10.4236/ojepi.2014.43021 
[9] Bankole, A., et al. (2007) Sexual Behavior, Knowledge and Information Sources of Very Young Adolescents in Four Sub-Saharan African Countries. African Journal of Reproductive Health, 11, 28-43. https://doi.org/10.2307/25549730

[10] Touré-Fall, A.O., Dièye, T.N.D., Sall, A., et al. (2009) Risque résiduel de transmission du VIH et du VHB par transfusion sanguine entre 2003 et 2005 au Centre national de transfusion sanguine de Dakar (Sénégal). Transfusion Clinique et Biologique, 16, 439-443. https://doi.org/10.1016/j.tracli.2009.09.005

[11] Pillonel, J. Surveillance épidémiologique des donneurs de sang et risque résiduel de transmission du VIH, de l'HTLV, du VHC et du VHB par transfusion en France entre 2008 et 2010 .

[12] Nagalo, M.B., Sanou, M., Bisseye, C., et al. (2011) Seroprevalence of Human Immunodeficiency Virus, Hepatitis B and C Viruses and Syphilis among Blood Donors in Koudougou (Burkina Faso) in 2009. Blood Transfusion, 9, 419-424.

[13] Mwenebitu David, L., Mabaya Gael, B., Christian, P., et al. (2018) Risk Factors of the Transmission of the HIV, Hepatitis B and C and Syphilis among Blood Donors at the Saint Luc General Hospital of Kisantu, Democratic Republic of Congo. Journal of HIV \& Retro Virus, 4, 18-21. https://doi.org/10.21767/2471-9676.100050

[14] Organisation Mondiale de la Santé (2011) Vers 100\% de dons volontaires. Cadre Mondial d'action. Editions de l'OMS. Génève. 